\title{
Generalized Balanced Tournament Designs with Block Size Four*
}

\author{
Yeow Meng Chee Han Mao Kiah \\ School of Physical and Mathematical Sciences \\ Nanyang Technological University \\ Singapore 637371 \\ \{YMChee,KIAH0001\}@ntu.edu.sg
}

\author{
Chengmin Wang ${ }^{\dagger}$ \\ School of Science \\ Jiangnan University \\ Wuxi, China 214122 \\ wcm@jiangnan.edu.cn
}

Submitted: Sep 16, 2012; Accepted: Jun 1, 2013; Published: Jun 7, 2013

Mathematics Subject Classifications: 05B05, 94B25

\begin{abstract}
In this paper, we remove the outstanding values $m$ for which the existence of a $\operatorname{GBTD}(4, m)$ has not been decided previously. This leads to a complete solution to the existence problem regarding $\operatorname{GBTD}(4, m) \mathrm{s}$.
\end{abstract}

Keywords: generalized balanced tournament design; holey generalized balanced tournament design; starter-adder

\section{Introduction}

A set system is a pair $\mathfrak{S}=(X, \mathcal{B})$, where $X$ is a finite set of points and $\mathcal{B}$ is a collection of subsets of $X$. Elements of $\mathcal{B}$ are called blocks. The order of $\mathfrak{S}$ is $|X|$, the number of points. Let $K$ be a set of positive integers. A set system $(X, \mathcal{B})$ is said to be $K$-uniform if $|B| \in K$ for all $B \in \mathcal{B}$. Let $(X, \mathcal{B})$ be a set system and $S \subseteq X$. A partial $\alpha$-parallel class over $X \backslash S$ of $(X, \mathcal{B})$ is a set of blocks $\mathcal{A} \subseteq \mathcal{B}$ such that each point of $X \backslash S$ occurs in exactly $\alpha$ blocks of $\mathcal{A}$, and each point of $S$ occurs in no block of $\mathcal{A}$. A partial $\alpha$-parallel class over $X$ is simply called an $\alpha$-parallel class. We adopt the convention that if $\alpha$ is not specified, then it is taken to be one, so that a parallel class refers to a 1-parallel class. A set system $(X, \mathcal{B})$ is said to be resolvable if $\mathcal{B}$ can be partitioned into parallel classes.

${ }^{*}$ Research of Y. M. Chee, H. M. Kiah, and C. Wang is supported in part by the Singapore National Research Foundation under Research Grant NRF-CRP2-2007-03. Research of C. Wang is also supported in part by NSFC under Grants No. 11271280 and 10801064.

${ }^{\dagger}$ Corresponding author. 
A balanced incomplete block design of order $v$, block size $k$, and index $\lambda$, denoted by $(v, k, \lambda)$-BIBD, is a $\{k\}$-uniform set system $(X, \mathcal{B})$ of order $v$ such that every 2-subset of $X$ is contained in precisely $\lambda$ blocks of $\mathcal{B}$. A resolvable $(k m, k, k-1)$-BIBD $(X, \mathcal{B})$ is called a generalized balanced tournament design (GBTD), or simply a $\operatorname{GBTD}(k, m)$, if the $m(k m-1)$ blocks of $\mathcal{B}$ are arranged in an $m \times(k m-1)$ array such that

(i) the set of blocks in each column is a parallel class, and

(ii) each point of $X$ is contained in at most $k$ cells of each row.

GBTDs were introduced by Lamken [3] and are useful in the construction of many combinatorial designs, including resolvable, near-resolvable, doubly resolvable, and doubly nearresolvable balanced incomplete block designs (see [2,3]). More recently, GBTDs have also found applications in near constant-composition codes [12], and codes for power line communications [1].

Schellenberg et al. [8] showed that a $\operatorname{GBTD}(2, m)$ exists for all positive integers $m \neq 2$. Lamken [4] showed that a $\operatorname{GBTD}(3, m)$ exists for all positive integers $m \neq 2$. For $k=4$, Yin et al. [12] obtained the following.

Theorem 1 (Yin et al. [12]). A $\operatorname{GBTD}(4, m)$ exists for all positive integers $m \geqslant 5$, except possibly when $m \in\{28,32,33,34,37,38,39,44\}$.

The purpose of this paper is to remove all the remaining eight possible exceptions in Theorem 1 and to show that a $\operatorname{GBTD}(4, m)$ exists for $m=4$ but not for $m \in\{2,3\}$.

Theorem 2. For each $m \in\{4,28,32,33,34,37,38,39,44\}$, a $\operatorname{GBTD}(4, m)$ exists. For $m=2$ and $3, a \operatorname{GBTD}(4, m)$ does not exist.

A $\operatorname{GBTD}(4,1)$ exists trivially. Combining Theorem 1 and Theorem 2, the existence of $\operatorname{GBTD}(4, m)$ is now completely determined.

Theorem 3. $A \operatorname{GBTD}(4, m)$ exists if and only if $m \geqslant 1$ and $m \neq 2,3$.

We first present a non-existence result.

Proposition 1.1. A $\operatorname{GBTD}(k, 2)$ does not exist for all $k \geqslant 2$.

Proof: Suppose $(X, \mathcal{B})$ is a $(2 k, k, k-1)$-BIBD whose blocks are organized into a $2 \times(2 k-1)$ array to form a $\operatorname{GBTD}(k, 2)$. Since $(X, \mathcal{B})$ is a $(2 k, k, k-1)$-BIBD, each point in $X$ appears in exactly $2 k-1$ blocks, and hence each point must appear either $k$ times in the first row and $k-1$ times in the second row, or vice versa.

Consider a point $x \in X$ that appears $k$ times in the first row and $k-1$ times in the second row. Let $y \in X$ be distinct from $x$. The cells in the first row can be classified as follows:

(i) Type- $x y$ : a cell that contains both $x$ and $y$. 
(ii) Type- $x \bar{y}$ : a cell that contains $x$ but not $y$.

(iii) Type- $\bar{x} y$ : a cell that contains $y$ but not $x$.

(iv) Type- $\bar{x} \bar{y}$ : a cell that contains neither $x$ nor $y$.

Let $\alpha$ and $\beta$ be the number of type- $x y$ cells and type- $\bar{x} y$ cells in the first row, respecitvely. Then we have the table

$\mathrm{T} 1=$\begin{tabular}{|r|c|c|c|c|}
\hline & Type- $x y$ & Type $-x \bar{y}$ & Type- $\bar{x} y$ & Type- $\bar{x} \bar{y}$ \\
\hline \# cells in first row & $\alpha$ & $k-\alpha$ & $\beta$ & $k-1-\beta$ \\
\hline \# cells in second row & $k-1-\beta$ & $\beta$ & $k-\alpha$ & $\alpha$ \\
\hline
\end{tabular}

where the second row is obtained from the first by the following observation: if a cell is of type- $x y$ (respectively, type- $x \bar{y}$, type- $\bar{x} y$, type- $\bar{x} \bar{y}$ ) in the first row, then the cell in the second row of the corresponding column is of type- $\bar{x} \bar{y}$ (respectively, type- $\bar{x} y$, type- $x \bar{y}$, type- $x y$ ). On the other hand, the total number of type- $x y$ cells is $k-1$, since $(X, \mathcal{B})$ is a BIBD of index $k-1$. Hence, we have $\alpha+(k-1-\beta)=k-1$, implying $\alpha=\beta$.

Considering the number of occurrences of $y$ in the first row and the number of occurrences of $y$ in the second row of the GBTD give the inequalities

$$
\begin{aligned}
\alpha+\beta & \leqslant k, \\
2 k-1-\alpha-\beta & \leqslant k
\end{aligned}
$$

from which, and $\alpha=\beta$ shown earlier, follow that

$$
\alpha=\lfloor k / 2\rfloor
$$

Table T1 can therefore be revised to

$\mathrm{T} 2=$\begin{tabular}{|r|c|c|c|c|}
\hline & Type- $x y$ & Type- $x \bar{y}$ & Type- $\bar{x} y$ & Type- $\bar{x} \bar{y}$ \\
\hline \# cells in first row & $\lfloor k / 2\rfloor$ & $\lceil k / 2\rceil$ & $\lfloor k / 2\rfloor$ & $\lceil k / 2\rceil-1$ \\
\hline \# cells in second row & $\lceil k / 2\rceil-1$ & $\lfloor k / 2\rfloor$ & $\lceil k / 2\rceil$ & $\lfloor k / 2\rfloor$ \\
\hline
\end{tabular}

Counting in two ways the number of elements in the set

$$
\{(y, C): y \in X, y \neq x \text {, and } C \text { is a cell of type- } x y \text { in the second row }\} \text {. }
$$

gives

$$
(2 k-1)(\lceil k / 2\rceil-1)=(k-1)^{2},
$$

which is a contradiction. 


\section{Existence of $\operatorname{GBTD}(4, m)$ s with $m=3$ and 4}

For a positive integer $n$, the set $\{1,2, \ldots, n\}$ is denoted by $[n]$. Let $\Sigma$ be a set of $q$ symbols. A q-ary code of length $n$ over $\Sigma$ is a subset $\mathcal{C} \subseteq \Sigma^{n}$. Elements of $\mathcal{C}$ are called codewords. The size of $\mathcal{C}$ is the number of codewords in $\mathcal{C}$. For $i \in[n]$, the $i$ th coordinate of a codeword $\mathrm{u} \in \mathcal{C}$ is denoted $\mathrm{u}_{i}$, so that $\mathrm{u}=\left(\mathrm{u}_{1}, \mathrm{u}_{2}, \ldots, \mathrm{u}_{n}\right)$.

The symbol weight of $\mathbf{u} \in \Sigma^{n}$, denoted $\operatorname{swt}(\mathbf{u})$, is the maximum frequency of appearance of a symbol in $u$, that is,

$$
\operatorname{swt}(\mathbf{u})=\max _{\sigma \in \Sigma}\left|\left\{\mathbf{u}_{i}=\sigma: i \in[n]\right\}\right|
$$

A code has constant symbol weight $w$ if all of its codewords have symbol weight exactly $w$. The (Hamming) distance between $\mathrm{u}, \mathrm{v} \in \Sigma^{n}$ is $d_{\mathrm{H}}(\mathrm{u}, \mathrm{v})=\left|\left\{i \in[n]: \mathrm{u}_{i}=\mathrm{v}_{i}\right\}\right|$, the number of coordinates at which $\mathrm{u}$ and $\mathrm{v}$ differ. A code $\mathcal{C}$ is said to have distance $d$ if $d_{\mathrm{H}}(\mathrm{u}, \mathrm{v}) \geqslant d$ for all distinct $\mathrm{u}, \mathrm{v} \in \mathcal{C}$. A $q$-ary code of length $n$, constant symbol weight $w$, and distance $d$ is referred to as an $(n, d, w)_{q}$-symbol weight code. An $(n, d, w)_{q}$-symbol weight code with maximum size is said to be optimal.

Chee et al. [1] established the following relation between a GBTD and a symbol weight code.

Theorem 4 (Chee et al. [1]). A $\operatorname{GBTD}(k, m)$ exists if and only if an optimal $(k m-1, k(m-$ $1), k)_{m}$-symbol weight code exists.

In view of Theorem 4 , to prove the nonexistence of a $\operatorname{GBTD}(4,3)$, it suffices to show that there does not exist a ternary code of length 11, constant symbol weight four, and size 12, that is of equidistance eight. Consider the Gilbert graph $G=(V, E)$, where $V=\left\{\mathrm{u} \in[3]^{11}\right.$ : $\operatorname{swt}(\mathbf{u})=4\}$ and two vertices $\mathrm{u}, \mathrm{v} \in V$ are adjacent in $G$ if and only if $d_{\mathrm{H}}(\mathrm{u}, \mathbf{v})=8$. Then there exists a ternary code of length 11 , constant symbol weight four, and size 12, that is of equidistance eight if and only if there exists a clique of size $12 \mathrm{in} G$. It is not hard to see that $G$ is vertex-transitive so that we can just search for a clique of size 11 in $G^{\prime}$, the subgraph of $G$ induced by the set of vertices $\left\{\mathrm{v} \in V: d_{\mathrm{H}}(\mathrm{u}, \mathrm{v})=8\right\}$ for some fixed $\mathrm{u} \in V$. This induced subgraph $G^{\prime}$ has 8001 vertices and 7233060 edges. We solve this clique-finding problem using Cliquer, an implementation of Östergård's clique-finding algorithm by Niskanen and Östergård [6]. The result is that the largest clique in $G^{\prime}$ has size 10. Consequently, we have the following.

Proposition 2.1. There does not exist a $\operatorname{GBTD}(4,3)$.

There exists, however, a $\operatorname{GBTD}(4,4)$. Unfortunately, a $\operatorname{GBTD}(4,4)$ is too large to be found by the method of clique-finding above. Instead, to curb the search space, we assume the existence of some automorphisms acting on the $\operatorname{GBTD}(4,4)$ to be found. Let $(X, \mathcal{B})$ be a $\operatorname{GBTD}(4,4)$, where $X=\mathbb{Z}_{4} \times \mathbb{Z}_{4}$. If $B \subseteq X$ and $x \in X, B+x$ denotes the set $\{b+x: b \in B\}$. If $\mathrm{A}$ is an array over $X$ and $x \in X, \mathrm{~A}+x$ denotes the array obtained by adding $x$ to every entry of $A$. For succinctness, a point $(x, y) \in \mathbb{Z}_{4} \times \mathbb{Z}_{4}$ is sometimes written $x y$. 
The $\operatorname{GBTD}(4,4)$ we construct contains the $4 \times 3$ subarray

$$
A_{0}=\begin{array}{llll}
\{00,02,20,22\} & \{11,13,31,33\} & \{10,12,30,32\} \\
\{01,03,21,23\} & \{00,02,20,22\} & \{11,13,31,33\} \\
\{10,12,30,32\} & \{01,03,21,23\} & \{00,02,20,22\} \\
\{11,13,31,33\} & \{10,12,30,32\} & \{01,03,21,23\}
\end{array} .
$$

The blocks in $\mathrm{A}_{0}$ contain exactly the 2-subsets $\{a b, c d\} \subseteq X$, where $a+c \equiv b+d \equiv 0 \bmod 2$, each thrice. The remaining $4 \times 12$ subarray of the $\operatorname{GBTD}(4,4)$ is built from a set of 12 base blocks $\mathcal{S}=\left\{B_{i, j}: i \in[3]\right.$ and $\left.0 \leqslant j \leqslant 3\right\}$ as follows. Let

$$
\mathrm{A}_{1}=\begin{array}{lll}
B_{1,0} & B_{2,0} & B_{3,0} \\
B_{1,1} & B_{2,1} & B_{3,1} \\
B_{1,2} & B_{2,2} & B_{3,2} \\
B_{1,3} & B_{2,3} & B_{3,3}
\end{array}
$$

Then the $4 \times 12$ subarray is given by

$$
\begin{array}{llll}
\mathrm{A}_{1} & \mathrm{~A}_{1}+(0,1) & \mathrm{A}_{1}+(0,2) & \mathrm{A}_{1}+(0,3) .
\end{array} .
$$

For

$$
\begin{array}{lllll}
\mathrm{A}_{0} & \mathrm{~A}_{1} & \mathrm{~A}_{1}+(0,1) & \mathrm{A}_{1}+(0,2) & \mathrm{A}_{1}+(0,3) \\
\hline
\end{array}
$$

to be a $\operatorname{GBTD}(4,4)$, the following conditions are imposed:

(i) $\bigcup_{j=0}^{3} B_{i, j}=\mathbb{Z}_{4} \times \mathbb{Z}_{4}$, for $i \in[3]$, so that every column is a parallel class.

(ii) For each $j, 0 \leqslant j \leqslant 3$, each element of $\mathbb{Z}_{4}$ appears exactly three times as a first coordinate among the elements of $\bigcup_{i=1}^{3} B_{i, j}$, so that every row contains each element of $\mathbb{Z}_{4} \times \mathbb{Z}_{4}$ at most three times.

(iii) Let $k, l \in \mathbb{Z}_{4}$ and define $\Delta_{k, l} \mathcal{S}$ to be the multiset $\bigcup_{A \in \mathcal{S}}\{x-y:(k, x),(l, y) \in A\}$. Then

$$
\Delta_{k, l} \mathcal{S}= \begin{cases}\{1,1,1,3,3,3\}, & \text { if } k=l \text { or } k+l \equiv 0 \bmod 2 \\ \{0,0,0,1,1,1,2,2,2,3,3,3\}, & \text { otherwise }\end{cases}
$$

This ensures that every 2-subset of $X$ not contained in any block in $A_{0}$ is contained in exactly three blocks in $A_{1}, A_{1}+(0,1), A_{1}+(0,2)$, or $A_{1}+(0,3)$.

A computer search found the following array $A_{1}$ that satisfies all the conditions above.

$$
A_{1}=\begin{array}{llll}
\{23,22,32,11\} & \{10,00,21,11\} & \{00,01,30,33\} \\
\{20,01,30,33\} & \{33,02,03,12\} & \{10,13,22,23\} \\
\{31,00,12,21\} & \{01,13,20,32\} & \{02,11,21,32\} \\
\{02,10,13,03\} & \{22,23,30,31\} & \{03,12,20,31\}
\end{array} .
$$

Consequently, we have the following.

Proposition 2.2. There exists a $\operatorname{GBTD}(4,4)$. 


\section{Incomplete Holey GBTDs}

Let $(X, \mathcal{B})$ be a set system, and let $\mathcal{G}$ be a partition of $X$ into subsets, called groups. The triple $(X, \mathcal{G}, \mathcal{B})$ is a group divisible design (GDD) of index $\lambda$ when every 2-subset of $X$ not contained in a group appears in exactly $\lambda$ blocks, and $|B \cap G| \leqslant 1$ for all $B \in \mathcal{B}$ and $G \in \mathcal{G}$. We denote a $\operatorname{GDD}(X, \mathcal{G}, \mathcal{B})$ of index $\lambda$ by $(K, \lambda)$-GDD if $(X, \mathcal{B})$ is $K$-uniform. The type of a $\operatorname{GDD}(X, \mathcal{G}, \mathcal{B})$ is the multiset $[|G|: G \in \mathcal{G}]$. When more convenient, the exponential notation is used to describe the type of a GDD: a GDD of type $g_{1}^{t_{1}} g_{2}^{t_{2}} \cdots g_{s}^{t_{s}}$ is a GDD where there are exactly $t_{i}$ groups of size $g_{i}, i \in[s]$.

Suppose further $\mathcal{G}=\left\{G_{1}, G_{2}, \ldots G_{s}\right\}$ and $\mathcal{H}=\left\{H_{1}, H_{2}, \ldots H_{s}\right\}$ is a collection of subsets of $X$ with the property $H_{i} \subseteq G_{i}, 0 \leqslant i \leqslant s$. Let $H=\bigcup_{i=1}^{s} H_{i}$. Then the quadruple $(X, \mathcal{G}, \mathcal{H}, \mathcal{B})$ is an incomplete group divisible design (IGDD) of index $\lambda$ when every 2 -subset of $X$ not contained in a group or $H$ appears in exactly $\lambda$ blocks, and $|B \cap G| \leqslant 1$ and $|B \cap H| \leqslant 1$ for all $B \in \mathcal{B}$ and $G \in \mathcal{G}$. The type of an $\operatorname{IGDD}\left(X,\left\{G_{1}, G_{2}, \ldots, G_{s}\right\},\left\{H_{1}, H_{2}, \ldots, H_{s}\right\}, \mathcal{B}\right)$ is the multiset $\left[\left(\left|G_{i}\right|,\left|H_{i}\right|\right): 1 \leqslant i \leqslant s\right]$ and we use the exponential notation when more convenient.

Let $k, g, u$, and $w$ be positive integers such that $k \mid g$ and $u \geqslant(k+1) w$. Let $R_{i}=\{r \in \mathbb{Z}$ : $i g / k \leqslant r \leqslant(i+1) g / k-1\}$. An incomplete holey GBTD with block size $k$ and type $g^{(u, w)}$, denoted $\operatorname{IHGBTD}\left(k, g^{(u, w)}\right)$, is a $(\{k\}, k-1)$-IGDD $\left(X,\left\{G_{0}, G_{1}, \ldots, G_{u-1}\right\},\left\{\varnothing, \ldots, \varnothing, G_{u-w}\right.\right.$, $\left.\left.\ldots, G_{u-1}\right\}, \mathcal{B}\right)$ of type $(g, 0)^{u-w}(g, g)^{w}$, whose blocks are arranged in a $(g u / k) \times g(u-1)$ array $A$, with rows and columns indexed by elements from the sets $\{0,1, \ldots, g u / k-1\}$ and $\{0,1, \ldots, g(u-1)-1\}$, respectively, such that the following properties are satisfied.

(i) The $(g w / k) \times g(w-1)$ subarray whose rows are indexed by $r \in R_{i}$, where $u-w \leqslant i \leqslant$ $u-1$, and columns indexed by $c$, where $g(u-w) \leqslant c \leqslant g(u-1)-1$, is empty.

(ii) For each $i, 0 \leqslant i \leqslant u-w-1$, the blocks in row $r \in R_{i}$ form a partial $k$-parallel class over $X \backslash G_{i}$, and for each $i, u-w \leqslant i \leqslant u-1$, the blocks in row $r \in R_{i}$ form a partial $k$-parallel class over $X \backslash\left(\bigcup_{j=u-w}^{w-1} G_{j}\right)$.

(iii) For each $j, 0 \leqslant j \leqslant g(u-w)-1$, the blocks in column $j$ form a parallel class, and for each $j, g(u-w) \leqslant j \leqslant g(u-1)-1$, the blocks in column $j$ form a partial parallel class over $X \backslash\left(\bigcup_{i=u-w}^{w-1} G_{j}\right)$.

Each group acts as a hole of the design, since no block contains any 2-subset of a group. The design is also incomplete in the sense that the array A contains an empty $(g w / k) \times g(w-1)$ subarray.

We note that an $\operatorname{IHGBTD}\left(k, g^{(u, 1)}\right)$ is a holey $\operatorname{GBTD}, \operatorname{HGBTD}\left(k, g^{u}\right)$, as defined by Yin et al. [12]. The following was established by Yin et al. [12].

Proposition 3.1 (Yin et al. [12]). If there exists an $\operatorname{HGBTD}\left(k, k^{u}\right)$, then there exists a $\operatorname{GBTD}(k, u)$.

Proposition 3.1 shows that a $\operatorname{GBTD}(k, u)$ can be obtained from an $\operatorname{HGBTD}\left(k, k^{u}\right)$. The next result shows how we can obtain an $\operatorname{HGBTD}\left(k, g^{u}\right)$ (and, in particular, an $\operatorname{HGBTD}\left(k, k^{u}\right)$ from an $\operatorname{IHGBTD}\left(k, g^{(u, w)}\right)$ and an $\operatorname{HGBTD}\left(k, g^{w}\right)$. 
Proposition 3.2. If there exist an $\operatorname{IHGBTD}\left(k, g^{(u, w)}\right)$ and an $\operatorname{HGBTD}\left(k, g^{w}\right)$, then there exists an $\operatorname{HGBTD}\left(k, g^{u}\right)$.

Proof: When $w=1$, an $\operatorname{HGBTD}\left(k, g^{w}\right)$ is empty and an $\operatorname{IHGBTD}\left(k, g^{(u, w)}\right)$ is just an $\operatorname{HGBTD}\left(k, g^{u}\right)$. So assume $w>1$ and let $(X, \mathcal{G}, \mathcal{B})$ be an $\operatorname{IHGBTD}\left(k, g^{(u, w)}\right)$ with $\mathcal{G}=$ $\left\{G_{0}, G_{1}, \ldots, G_{u-1}\right\}$. Fill in the empty subarray of this $\operatorname{IHGBTD}$ with an $\operatorname{HGBTD}\left(k, g^{w}\right)$, $\left(X^{\prime}, \mathcal{G}^{\prime}, \mathcal{B}^{\prime}\right)$, with $\mathcal{G}^{\prime}=\left\{G_{u-w}, G_{u-w+1}, \ldots, G_{u-1}\right\}$ and $X^{\prime}=\bigcup_{i=u-w}^{u-1} G_{i}$. The resulting array is a $\operatorname{HGBTD}\left(k, g^{u}\right),\left(X, \mathcal{G}, \mathcal{B} \cup \mathcal{B}^{\prime}\right)$.

\section{Starter-Adder Construction for IHGBTD}

The starter-adder technique first used by Mullin and Nemeth [5] to construct Room squares (also a combinatorial array) has been useful in constructing many types of designs with orthogonality properties, including GBTDs (see $[3,7,10,11,12]$ ). Here, we extend the technique to the construction of IHGBTDs. Since only $\operatorname{IHGBTD}\left(k, g^{(u, w)}\right)$ with $g=k$ are considered here, we describe our construction for this case.

Let $\Gamma$ be an additive abelian group of order $k(u-w)$ with $u \geqslant(k+1) w$, and let $\Gamma_{0} \subseteq \Gamma$ be a subgroup of order $k$. Fix a set, $\Delta=\left\{\delta_{0}=0, \delta_{1}, \ldots, \delta_{u-w-1}\right\} \subseteq \Gamma$, of representatives for the cosets of $\Gamma_{0}$ so that $\Gamma_{i}=\Gamma_{0}+\delta_{i}, 0 \leqslant i \leqslant u-w-1$, are the cosets of $\Gamma_{0}$. Let $H$ be a set of $k w$ points such that $H$ and $\Gamma$ are disjoint. Further, let $H$ be partitioned into $w$ subsets $H_{0}, H_{1}, \ldots, H_{w-1}$ of size $k$ each.

We take $X=\Gamma \bigcup H$ to be the point set of an $\operatorname{IHGBTD}\left(k, k^{(u, w)}\right)$. An intransitive starter for an $\operatorname{IHGBTD}\left(k, k^{(u, w)}\right)$, with groups $\left\{G_{0}, G_{1}, \ldots, G_{u-1}\right\}$, where

$$
G_{i}= \begin{cases}\Gamma_{i}, & \text { if } 0 \leqslant i \leqslant u-w-1 \\ H_{i-u+w}, & \text { if } u-w \leqslant i \leqslant u-1,\end{cases}
$$

is defined as a quadruple $(X, \mathcal{S}, \mathcal{R}, \mathcal{C})$ satisfying the properties:

(i) $(X, \mathcal{S}),(X, \mathcal{R})$, and $(X, \mathcal{C})$ are $\{k\}$-uniform set systems of size $u-w$, w, and $w-1$, respectively;

(ii) among the blocks in $\mathcal{S}, k w$ of them intersects $H$ in one point, that is, $\mid\{B \in \mathcal{S}$ : $|B \cap H|=1\} \mid=k w$;

(iii) blocks in $\mathcal{R}$ are each disjoint from $H$;

(iv) blocks in $\mathcal{C}$ are each disjoint from $H$, and $\bigcup_{i=0}^{u-w-1}\left(\delta_{i}+C\right)=\Gamma$, for each $C \in \mathcal{C}$.

(v) $\mathcal{S} \cup \mathcal{R}$ is a partition of $X$;

(vi) the difference list from the base blocks of $\mathcal{S} \cup \mathcal{R} \bigcup \mathcal{C}$ contains every element of $\Gamma \backslash \Gamma_{0}$ precisely $k-1$ times, and no element in $\Gamma_{0}$. 
Suppose $\mathcal{S}=\left\{B_{0}, B_{1}, \ldots, B_{u-w-1}\right\}$. Then a corresponding adder $\Omega(\mathcal{S})$ for $\mathcal{S}$ is a permutation $\Omega(\mathcal{S})=\left(\omega_{0}, \omega_{1}, \ldots, \omega_{u-w-1}\right)$ of the $u-w$ elements of the representative system $\Delta$ satisfying the following property:

(vii) the multiset $\left(\bigcup_{i=0}^{u-w-1}\left(B_{i}+\omega_{i}\right)\right) \bigcup\left(\bigcup_{C \in \mathcal{C}} C\right)$ contains exactly $k$ elements (not necessarily distinct) from $\Gamma_{j}$ for $1 \leqslant j \leqslant u-w-1$, and is disjoint from $\Gamma_{0}$. We remark that when $B \in \mathcal{S}$ and $B \cap H=\{\infty\}$, or $B=\left\{\infty, b_{1}, b_{2}, \ldots, b_{k-1}\right\}$, the block $B+\gamma$ is defined to be $\left\{\infty, b_{1}+\gamma, b_{2}+\gamma, \ldots, b_{k-1}+\gamma\right\}$ for $\gamma \in \Gamma$.

The result below shows how to construct an IHGBTD from an intransitive starter and its corresponding adder.

Proposition 4.1. Let $\Gamma$ be an additive abelian group of order $k(u-w)$ with $u \geqslant(k+1) w$ and $\Gamma_{0}$ be a subgroup of order $k$. Define $X$ and the groups $G_{i}(0 \leqslant i \leqslant u-1)$ as above. If there exists an intransitive starter $(X, \mathcal{S}, \mathcal{R}, \mathcal{C})$ with groups $\left\{G_{i}: 0 \leqslant i \leqslant u-1\right\}$, a corresponding adder $\Omega(\mathcal{S})$, then there exists an $\operatorname{IHGBTD}\left(k, k^{(u, w)}\right)$.

Proof: Retain the notations in the definition of intransitive starter and adder. Suppose

$$
\mathcal{A}=\{A+\gamma: \gamma \in \Gamma, A \in \mathcal{S} \cup \mathcal{R} \cup \mathcal{C}\}
$$

then $\left(X,\left\{G_{0}, G_{1}, \ldots, G_{u-1}\right\},\left\{\varnothing, \ldots, \varnothing, H_{0}, \ldots, H_{w-1}\right\}, \mathcal{A}\right)$ forms a $(\{k\}, k-1)$-IGDD of type $(k, 0)^{u-w}(k, k)^{w}$ by Condition (vi) in the definition of intransitive starter. Therefore, it remains to arrange the blocks in a $u \times k(u-1)$ array.

First, consider the blocks $\mathcal{S}$. Consider a $(u-w) \times(u-w)$ array $\mathbf{S}$, whose rows and columns are indexed with the elements in $\Delta$. Now identify the elements in $\Delta$ with elements in the quotient group $\Gamma / \Gamma_{0}$, so that the binary operation $\stackrel{\circ}{+}$ on $\Delta$ is defined by the additive operation on $\Gamma / \Gamma_{0}$. In addition, for $\delta \in \Delta$, denote the additive inverse of $\delta$ by $\stackrel{\circ}{-} \delta$. That is, $\delta \stackrel{\circ}{+}(\stackrel{\circ}{-\delta})=\delta_{0}$.

So, for $0 \leqslant i, j \leqslant u-w-1$, we place the block $B_{i}+\delta_{j}$ at the cell $\left(\delta_{j} \stackrel{\circ}{-} \delta_{l}, \delta_{j}\right)$ if $\delta_{l}=\omega_{i}$. Note that this placement is well defined because $\Omega(\mathcal{S})$ is a permutation of $\Delta$. Let $\Gamma_{0}=\left\{\gamma_{0}=\right.$ $\left.0, \gamma_{1}, \cdots, \gamma_{k-1}\right\}$. Form a $(u-w) \times k(u-w)$ array $\widehat{S}$ from the square $\mathrm{S}$ by concatenating $k$ squares $\mathrm{D}+\gamma_{i}$ where $0 \leqslant i \leqslant k-1$ as follows.

$$
\widehat{\mathrm{S}}=\begin{array}{llll|}
\mathrm{S} & \mathrm{S}+\gamma_{1} & \cdots & \mathrm{S}+\gamma_{k-1} \\
\hline
\end{array}
$$

Next, let $\mathcal{R}=\left\{R_{1}, R_{2}, \ldots, R_{w}\right\}$ and construct a $w \times k(u-w)$ array $\widehat{R}$ in the following way:

$$
\widehat{R}=\begin{array}{llll}
\mathrm{R} & \mathrm{R}+\gamma_{1} & \cdots & \mathrm{R}+\gamma_{k-1}
\end{array},
$$

where the $w \times w$ subarray $\mathrm{R}$ is given by

$$
\mathrm{R}=\begin{array}{cccc}
R_{1} & R_{1}+\delta_{1} & \cdots & R_{1}+\delta_{u-w-1} \\
R_{2} & R_{2}+\delta_{1} & \cdots & R_{2}+\delta_{u-w-1} \\
\vdots & \vdots & \ddots & \vdots \\
R_{w} & R_{w}+\delta_{1} & \cdots & R_{w}+\delta_{u-w-1} \\
\hline
\end{array}
$$


Similarly, let $\mathcal{C}=\left\{C_{0}, C_{1}, \ldots, C_{w-2}\right\}$, and we construct a $(u-w) \times k(w-1)$ array $\widehat{\mathrm{C}}$.

$$
\widehat{\mathrm{C}}=\begin{array}{llll}
\mathrm{C}_{0} & \mathrm{C}_{1} & \cdots & \mathrm{C}_{w-2} \\
\hline
\end{array}
$$

where each $(u-w) \times k$ subarray $\mathrm{C}_{i}, 0 \leqslant i \leqslant w-2$, is given by

$$
\mathrm{C}_{i}=\begin{array}{cccc|}
C_{i} & C_{i}+\gamma_{1} & \cdots & C_{i}+\gamma_{k-1} \\
C_{i}+\delta_{1} & C_{i}+\delta_{1}+\gamma_{1} & \cdots & C_{i}+\delta_{1}+\gamma_{k-1} \\
\vdots & \vdots & \ddots & \vdots \\
C_{i}+\delta_{u-w-1} & C_{i}+\delta_{u-w-1}+\gamma_{1} & \cdots & C_{i}+\delta_{u-w-1}+\gamma_{k-1} \\
\hline
\end{array}
$$

Finally, let

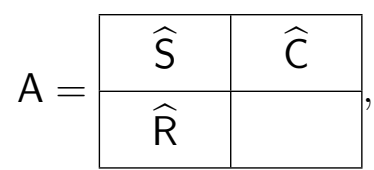

and it is readily verified that the placement results in an $\operatorname{IHGBTD}\left(k, k^{(u, w)}\right)$.

\section{Proof of Theorem 1.2}

We first remove all the eight remaining values in Theorem 1.

Lemma 5. For $(u, w) \in\{(28,5),(32,5),(33,6)\}$, an $\operatorname{IHGBTD}\left(4,4^{(u, w)}\right)$ exists.

Proof: We apply Proposition 4.1 to construct the desired IHGBTDs. Take

$$
\begin{aligned}
\Gamma & =\mathbb{Z}_{u-w} \times \mathbb{Z}_{4}, \\
\Gamma_{0} & =\{0\} \times \mathbb{Z}_{4}, \\
\Delta & =\{(0,0),(1,0), \ldots,(u-w-1,0)\}, \text { and } \\
H & =\bigcup_{i=0}^{w-1} H_{i}, \text { where } H_{i}=\left\{\infty_{i}, \infty_{i+w}, \infty_{i+2 w}, \infty_{i+3 w}\right\} \text { for } 0 \leqslant i \leqslant w-1 .
\end{aligned}
$$

For each pair $(u, w) \in\{(28,5),(32,5),(33,6)\}$, the desired intransitive starter and corresponding adder are displayed below. Here we write the element $(a, b)$ of $\Gamma$ as $a_{b}$ for succinctness.

When $(u, w)=(28,5)$ :

\begin{tabular}{lclclc}
\hline $\mathcal{S}$ & $\Omega(\mathcal{S})$ & $\mathcal{S}$ & $\Omega(\mathcal{S})$ & $\mathcal{S}$ & $\Omega(\mathcal{S})$ \\
\hline$\left\{4_{1}, 3_{0}, 7_{0}, 0_{0}\right\}$ & $17_{0}$ & $\left\{5_{0}, 19_{0}, 12_{1}, 1_{2}\right\}$ & $12_{0}$ & $\left\{18_{0}, 13_{3}, 16_{3}, 8_{1}\right\}$ & $19_{0}$ \\
$\left\{\infty_{0}, 3_{1}, 12_{2}, 11_{3}\right\}$ & $1_{0}$ & $\left\{\infty_{1}, 14_{3}, 6_{0}, 10_{3}\right\}$ & $21_{0}$ & $\left\{\infty_{2}, 14_{1}, 9_{1}, 20_{1}\right\}$ & $20_{0}$ \\
$\left\{\infty_{3}, 19_{1}, 10_{1}, 22_{2}\right\}$ & $7_{0}$ & $\left\{\infty_{4}, 3_{3}, 1_{3}, 2_{2}\right\}$ & $18_{0}$ & $\left\{\infty_{5}, 0_{2}, 15_{1}, 1_{0}\right\}$ & $15_{0}$ \\
$\left\{\infty_{6}, 1_{1}, 6_{3}, 9_{3}\right\}$ & $2_{0}$ & $\left\{\infty_{7}, 14_{0}, 11_{1}, 0_{1}\right\}$ & $10_{0}$ & $\left\{\infty_{8}, 0_{3}, 17_{2}, 21_{2}\right\}$ & $22_{0}$ \\
$\left\{\infty_{9}, 4_{3}, 8_{0}, 21_{0}\right\}$ & $6_{0}$ & $\left\{\infty_{10}, 13_{1}, 19_{3}, 16_{2}\right\}$ & $9_{0}$ & $\left\{\infty_{11}, 4_{2}, 21_{3}, 17_{1}\right\}$ & $5_{0}$ \\
$\left\{\infty_{12}, 17_{0}, 5_{2}, 21_{1}\right\}$ & $16_{0}$ & $\left\{\infty_{13}, 5_{1}, 20_{2}, 11_{2}\right\}$ & $4_{0}$ & $\left\{\infty_{14}, 22_{0}, 2_{3}, 16_{0}\right\}$ & $14_{0}$ \\
$\left\{\infty_{15}, 18_{3}, 20_{3}, 2_{0}\right\}$ & $0_{0}$ & $\left\{\infty_{16}, 12_{3}, 2_{1}, 22_{3}\right\}$ & $3_{0}$ & $\left\{\infty_{17}, 5_{3}, 7_{1}, 17_{3}\right\}$ & $8_{0}$ \\
$\left\{\infty_{18}, 6_{2}, 9_{0}, 19_{2}\right\}$ & $13_{0}$ & $\left\{\infty_{19}, 7_{2}, 8_{3}, 22_{1}\right\}$ & $11_{0}$ & & \\
\hline
\end{tabular}




$$
\begin{array}{rlrl}
\mathcal{C}= & \left\{18_{0}, 11_{1}, 5_{3}, 6_{2}\right\}, & \left\{18_{2}, 8_{3}, 19_{0}, 6_{1}\right\}, & \left\{14_{3}, 12_{0}, 3_{2}, 7_{1}\right\}, \\
\mathcal{R}= & \left\{5_{2}, 7_{1}, 16_{3}, 11_{0}\right\} . & & \\
\left\{3_{2}, 18_{2}, 16_{1}, 10_{2}\right\}, & \left\{8_{2}, 15_{0}, 20_{0}, 13_{2}\right\}, & \left\{13_{0}, 9_{2}, 18_{1}, 15_{3}\right\}, \\
& \left\{6_{1}, 7_{3}, 14_{2}, 15_{2}\right\}, & \left\{12_{0}, 10_{0}, 4_{0}, 11_{0}\right\} . &
\end{array}
$$

When $(u, w)=(32,5)$ :

\begin{tabular}{lclclc}
$\mathcal{S}$ & $\Omega(\mathcal{S})$ & $\mathcal{S}$ & $\Omega(\mathcal{S})$ & $\mathcal{S}$ & $\Omega(\mathcal{S})$ \\
\hline$\left\{4_{2}, 17_{2}, 16_{1}, 22_{2}\right\}$ & $16_{0}$ & $\left\{3_{1}, 4_{1}, 1_{0}, 9_{1}\right\}$ & $11_{0}$ & $\left\{4_{3}, 26_{3}, 22_{0}, 10_{3}\right\}$ & $0_{0}$ \\
$\left\{14_{1}, 6_{0}, 26_{0}, 3_{0}\right\}$ & $12_{0}$ & $\left\{\infty_{0}, 3_{3}, 24_{2}, 25_{1}\right\}$ & $7_{0}$ & $\left\{\infty_{1}, 2_{2}, 12_{0}, 1_{3}\right\}$ & $6_{0}$ \\
$\left\{\infty_{2}, 0_{1}, 26_{1}, 20_{2}\right\}$ & $4_{0}$ & $\left\{\infty_{3}, 25_{0}, 15_{0}, 23_{0}\right\}$ & $15_{0}$ & $\left\{\infty_{4}, 13_{0}, 21_{2}, 16_{0}\right\}$ & $3_{0}$ \\
$\left\{\infty_{5}, 5_{0}, 19_{3}, 12_{1}\right\}$ & $24_{0}$ & $\left\{\infty_{6}, 6_{3}, 14_{3}, 13_{2}\right\}$ & $1_{0}$ & $\left\{\infty_{7}, 1_{2}, 2_{0}, 0_{0}\right\}$ & $21_{0}$ \\
$\left\{\infty_{8}, 0_{2}, 10_{0}, 19_{0}\right\}$ & $14_{0}$ & $\left\{\infty_{9}, 15_{2}, 18_{2}, 0_{3}\right\}$ & $2_{0}$ & $\left\{\infty_{10}, 6_{1}, 5_{2}, 2_{3}\right\}$ & $17_{0}$ \\
$\left\{\infty_{11}, 12_{3}, 25_{2}, 11_{3}\right\}$ & $22_{0}$ & $\left\{\infty_{12}, 10_{1}, 21_{3}, 17_{3}\right\}$ & $18_{0}$ & $\left\{\infty_{13}, 17_{0}, 9_{0}, 20_{3}\right\}$ & $20_{0}$ \\
$\left\{\infty_{14}, 20_{0}, 3_{2}, 16_{3}\right\}$ & $5_{0}$ & $\left\{\infty_{15}, 12_{2}, 21_{1}, 8_{2}\right\}$ & $9_{0}$ & $\left\{\infty_{16}, 18_{1}, 11_{0}, 15_{3}\right\}$ & $10_{0}$ \\
$\left\{\infty_{17}, 1_{1}, 15_{1}, 17_{1}\right\}$ & $8_{0}$ & $\left\{\infty_{18}, 9_{2}, 16_{2}, 23_{2}\right\}$ & $13_{0}$ & $\left\{\infty_{19}, 14_{2}, 18_{3}, 21_{0}\right\}$ & $25_{0}$ \\
\hline
\end{tabular}

$$
\begin{array}{rlll}
\mathcal{C}= & \left\{1_{3}, 26_{0}, 16_{1}, 17_{2}\right\}, & \left\{5_{3}, 14_{1}, 24_{2}, 12_{0}\right\}, & \left\{19_{2}, 25_{0}, 17_{1}, 13_{3}\right\}, \\
& \left\{6_{2}, 8_{0}, 11_{3}, 13_{1}\right\} . & & \\
\mathcal{R}= & \left\{5_{1}, 11_{1}, 24_{3}, 20_{1}\right\}, & \left\{24_{1}, 18_{0}, 7_{0}, 6_{2}\right\}, & \left\{22_{1}, 25_{3}, 8_{0}, 13_{3}\right\}, \\
& \left\{19_{2}, 7_{2}, 2_{1}, 23_{3}\right\}, & \left\{7_{1}, 9_{3}, 26_{2}, 4_{0}\right\} . &
\end{array}
$$

When $(u, w)=(33,6)$ :

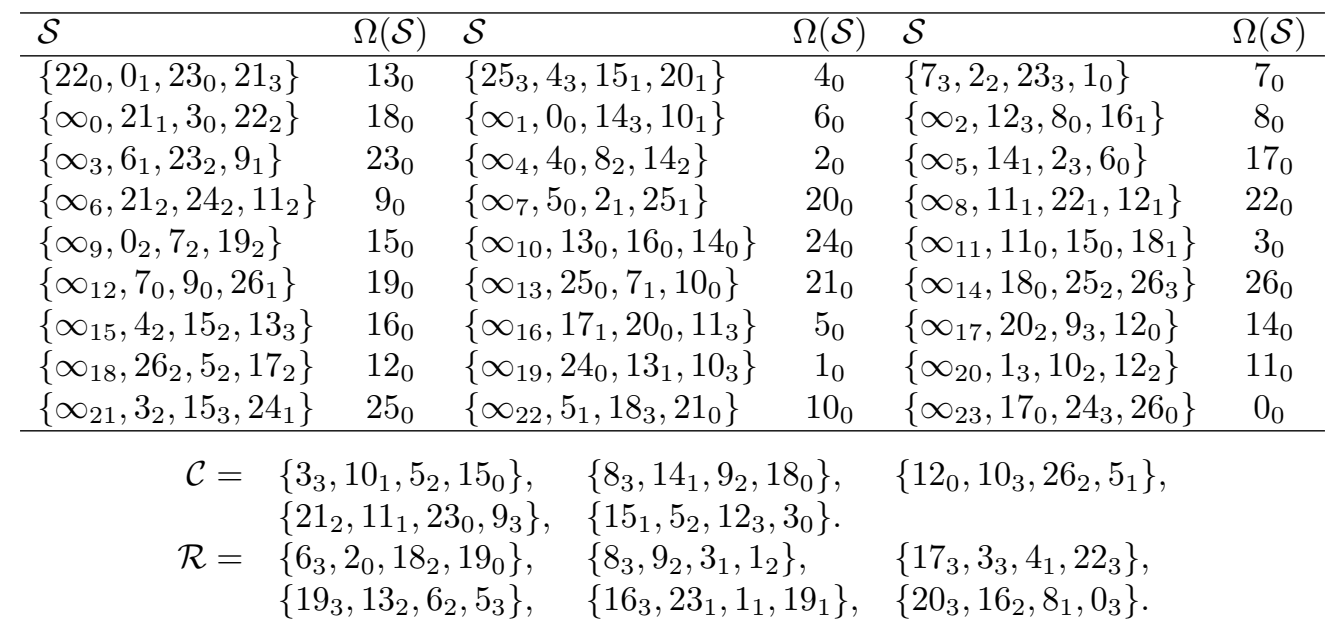

Lemma 6. For $(u, w) \in\{(34,6),(44,8)\}$, an $\operatorname{IHGBTD}\left(4,4^{(u, w)}\right)$ exists.

Proof: As with Lemma 5, we apply Proposition 4.1 to construct the desired IHGBTDs. Take

$$
\begin{aligned}
\Gamma & =\mathbb{Z}_{2(u-w)} \times \mathbb{Z}_{2}, \\
\Gamma_{0} & =\{0, u-w\} \times \mathbb{Z}_{2}, \\
\Delta & =\{(0,0),(1,0), \cdots,(u-w-1,0)\}, \text { and } \\
H & =\bigcup_{i=0}^{w-1} H_{i}, \text { where } H_{i}=\left\{\infty_{i}, \infty_{i+w}, \infty_{i+2 w}, \infty_{i+3 w}\right\} \text { for } 0 \leqslant i \leqslant w-1 .
\end{aligned}
$$


The desired intransitive starter and corresponding adder for $(u, w) \in\{(34,6),(44,8)\}$ are displayed below. Here we write the element $(a, b)$ of $\Gamma$ as $a_{b}$ for succinctness.

When $(u, w)=(34,6)$ :

\begin{tabular}{lclclc}
\hline $\mathcal{S}$ & $\Omega(\mathcal{S})$ & $\mathcal{S}$ & $\Omega(\mathcal{S})$ & $\mathcal{S}$ & $\Omega(\mathcal{S})$ \\
\hline$\left\{41_{1}, 16_{0}, 6_{0}, 15_{0}\right\}$ & $20_{0}$ & $\left\{36_{0}, 9_{0}, 33_{1}, 13_{1}\right\}$ & $16_{0}$ & $\left\{37_{0}, 18_{0}, 26_{1}, 4_{1}\right\}$ & $0_{0}$ \\
$\left\{16_{1}, 2_{1}, 4_{0}, 3_{1}\right\}$ & $3_{0}$ & $\left\{\infty_{0}, 20_{1}, 24_{0}, 42_{0}\right\}$ & $23_{0}$ & $\left\{\infty_{1}, 22_{1}, 30_{0}, 39_{1}\right\}$ & $11_{0}$ \\
$\left\{\infty_{2}, 14_{0}, 31_{1}, 1_{1}\right\}$ & $10_{0}$ & $\left\{\infty_{3}, 48_{0}, 45_{0}, 8_{0}\right\}$ & $25_{0}$ & $\left\{\infty_{4}, 25_{1}, 48_{1}, 14_{1}\right\}$ & $4_{0}$ \\
$\left\{\infty_{5}, 8_{1}, 30_{1}, 20_{0}\right\}$ & $12_{0}$ & $\left\{\infty_{6}, 6_{1}, 21_{0}, 44_{1}\right\}$ & $2_{0}$ & $\left\{\infty_{7}, 40_{1}, 33_{0}, 52_{1}\right\}$ & $1_{0}$ \\
$\left\{\infty_{8}, 45_{1}, 21_{1}, 28_{1}\right\}$ & $18_{0}$ & $\left\{\infty_{9}, 27_{0}, 28_{0}, 34_{1}\right\}$ & $17_{0}$ & $\left\{\infty_{10}, 42_{1}, 35_{1}, 37_{1}\right\}$ & $22_{0}$ \\
$\left\{\infty_{11}, 3_{0}, 22_{0}, 12_{0}\right\}$ & $19_{0}$ & $\left\{\infty_{12}, 44_{0}, 35_{0}, 39_{0}\right\}$ & $14_{0}$ & $\left\{\infty_{13}, 36_{1}, 7_{0}, 9_{1}\right\}$ & $7_{0}$ \\
$\left\{\infty_{14}, 15_{1}, 53_{1}, 51_{1}\right\}$ & $6_{0}$ & $\left\{\infty_{15}, 53_{0}, 11_{0}, 51_{0}\right\}$ & $15_{0}$ & $\left\{\infty_{16}, 50_{0}, 55_{1}, 10_{1}\right\}$ & $9_{0}$ \\
$\left\{\infty_{17}, 52_{0}, 32_{1}, 17_{1}\right\}$ & $13_{0}$ & $\left\{\infty_{18}, 55_{0}, 29_{1}, 25_{0}\right\}$ & $5_{0}$ & $\left\{\infty_{19}, 0_{1}, 7_{1}, 41_{0}\right\}$ & $27_{0}$ \\
$\left\{\infty_{20}, 12_{1}, 31_{0}, 47_{0}\right\}$ & $8_{0}$ & $\left\{\infty_{21}, 17_{0}, 27_{1}, 47_{1}\right\}$ & $21_{0}$ & $\left\{\infty_{22}, 19_{0}, 23_{0}, 29_{0}\right\}$ & $24_{0}$ \\
$\left\{\infty_{23}, 34_{0}, 40_{0}, 50_{1}\right\}$ & $26_{0}$ & & & & \\
\hline
\end{tabular}

$$
\begin{array}{rlll}
\mathcal{C}= & \left\{27_{1}, 10_{0}, 44_{1}, 51_{0}\right\}, & \left\{35_{1}, 15_{0}, 50_{0}, 14_{1}\right\}, & \left\{16_{1}, 51_{1}, 54_{0}, 27_{0}\right\}, \\
& \left\{24_{1}, 12_{0}, 37_{0}, 21_{1}\right\}, & \left\{39_{0}, 2_{1}, 45_{1}, 50_{0}\right\} . & \\
\mathcal{R}=\left\{13_{0}, 26_{0}, 38_{0}, 24_{1}\right\}, & \left\{54_{1}, 23_{1}, 46_{1}, 49_{1}\right\}, & \left\{1_{0}, 49_{0}, 18_{1}, 43_{0}\right\}, \\
& \left\{10_{0}, 2_{0}, 11_{1}, 54_{0}\right\}, & \left\{46_{0}, 19_{1}, 43_{1}, 5_{0}\right\}, & \left\{38_{1}, 32_{0}, 5_{1}, 0_{0}\right\} .
\end{array}
$$

When $(u, w)=(44,8)$ :

\begin{tabular}{lclclc}
\hline $\mathcal{S}$ & $\Omega(\mathcal{S})$ & $\mathcal{S}$ & $\Omega(\mathcal{S})$ & $\mathcal{S}$ & $\Omega(\mathcal{S})$ \\
\hline$\left\{32_{0}, 69_{1}, 36_{1}, 53_{1}\right\}$ & $20_{0}$ & $\left\{42_{1}, 65_{1}, 0_{0}, 43_{1}\right\}$ & $1_{0}$ & $\left\{39_{1}, 27_{1}, 45_{1}, 51_{1}\right\}$ & $3_{0}$ \\
$\left\{22_{1}, 39_{0}, 55_{1}, 33_{1}\right\}$ & $11_{0}$ & $\left\{\infty_{0}, 67_{0}, 40_{1}, 54_{0}\right\}$ & $22_{0}$ & $\left\{\infty_{1}, 23_{0}, 10_{1}, 34_{1}\right\}$ & $25_{0}$ \\
$\left\{\infty_{2}, 18_{0}, 67_{1}, 36_{0}\right\}$ & $28_{0}$ & $\left\{\infty_{3}, 25_{1}, 10_{0}, 28_{1}\right\}$ & $16_{0}$ & $\left\{\infty_{4}, 63_{1}, 6_{0}, 37_{0}\right\}$ & $29_{0}$ \\
$\left\{\infty_{5}, 16_{0}, 44_{0}, 2_{0}\right\}$ & $35_{0}$ & $\left\{\infty_{6}, 28_{0}, 50_{1}, 35_{1}\right\}$ & $10_{0}$ & $\left\{\infty_{7}, 43_{0}, 46_{1}, 32_{1}\right\}$ & $9_{0}$ \\
$\left\{\infty_{8}, 69_{0}, 52_{1}, 2_{1}\right\}$ & $13_{0}$ & $\left\{\infty_{9}, 37_{1}, 66_{0}, 71_{1}\right\}$ & $26_{0}$ & $\left\{\infty_{10}, 70_{1}, 21_{1}, 24_{1}\right\}$ & $8_{0}$ \\
$\left\{\infty_{11}, 71_{0}, 15_{1}, 47_{0}\right\}$ & $32_{0}$ & $\left\{\infty_{12}, 59_{0}, 19_{1}, 6_{1}\right\}$ & $23_{0}$ & $\left\{\infty_{13}, 9_{0}, 47_{1}, 20_{0}\right\}$ & $7_{0}$ \\
$\left\{\infty_{14}, 52_{0}, 46_{0}, 60_{1}\right\}$ & $24_{0}$ & $\left\{\infty_{15}, 17_{0}, 60_{0}, 22_{0}\right\}$ & $0_{0}$ & $\left\{\infty_{16}, 64_{0}, 54_{1}, 12_{0}\right\}$ & $17_{0}$ \\
$\left\{\infty_{17}, 49_{0}, 9_{1}, 53_{0}\right\}$ & $4_{0}$ & $\left\{\infty_{18}, 68_{0}, 0_{1}, 56_{1}\right\}$ & $15_{0}$ & $\left\{\infty_{19}, 27_{0}, 12_{1}, 4_{1}\right\}$ & $27_{0}$ \\
$\left\{\infty_{20}, 65_{0}, 68_{1}, 23_{1}\right\}$ & $2_{0}$ & $\left\{\infty_{21}, 20_{1}, 18_{1}, 8_{0}\right\}$ & $31_{0}$ & $\left\{\infty_{22}, 59_{1}, 17_{1}, 44_{1}\right\}$ & $14_{0}$ \\
$\left\{\infty_{23}, 1_{0}, 70_{0}, 26_{1}\right\}$ & $12_{0}$ & $\left\{\infty_{24}, 57_{1}, 11_{1}, 13_{0}\right\}$ & $21_{0}$ & $\left\{\infty_{25}, 16_{1}, 5_{0}, 7_{0}\right\}$ & $18_{0}$ \\
$\left\{\infty_{26}, 58_{1}, 4_{0}, 57_{0}\right\}$ & $5_{0}$ & $\left\{\infty_{27}, 41_{1}, 13_{1}, 31_{1}\right\}$ & $19_{0}$ & $\left\{\infty_{28}, 64_{1}, 56_{0}, 30_{1}\right\}$ & $30_{0}$ \\
$\left\{\infty_{29}, 19_{0}, 48_{0}, 21_{0}\right\}$ & $6_{0}$ & $\left\{\infty_{30}, 48_{1}, 58_{0}, 50_{0}\right\}$ & $33_{0}$ & $\left\{\infty_{31}, 40_{0}, 49_{1}, 5_{1}\right\}$ & $34_{0}$ \\
\hline
\end{tabular}

$$
\begin{aligned}
\mathcal{C}= & \left\{2_{1}, 3_{1}, 22_{0}, 69_{0}\right\}, & \left\{28_{1}, 69_{0}, 19_{1}, 62_{0}\right\}, & \left\{41_{1}, 4_{0}, 20_{1}, 59_{0}\right\}, \\
& \left\{57_{0}, 12_{1}, 4_{0}, 55_{1}\right\}, & \left\{41_{0}, 21_{1}, 32_{1}, 8_{0}\right\}, & \left\{7_{1}, 13_{0}, 14_{1}, 28_{0}\right\}, \\
& \left\{33_{1}, 21_{0}, 28_{1}, 52_{0}\right\} . & & \\
\mathcal{R}= & \left\{66_{1}, 3_{1}, 25_{0}, 29_{1}\right\}, & \left\{38_{0}, 34_{0}, 3_{0}, 24_{0}\right\}, & \left\{55_{0}, 15_{0}, 62_{0}, 45_{0}\right\}, \\
& \left\{62_{1}, 61_{0}, 42_{0}, 29_{0}\right\}, & \left\{51_{0}, 35_{0}, 30_{0}, 26_{0}\right\}, & \left\{61_{1}, 1_{1}, 14_{0}, 38_{1}\right\}, \\
& \left\{14_{1}, 11_{0}, 31_{0}, 63_{0}\right\}, & \left\{7_{1}, 33_{0}, 8_{1}, 41_{0}\right\} . &
\end{aligned}
$$

Lemma 7. For each $(u, w) \in\{(37,6),(38,7),(39,6)\}$, an $\operatorname{IHGBTD}\left(4,4^{(u, w)}\right)$ exists. 
When $(u, w)=(39,6)$ :

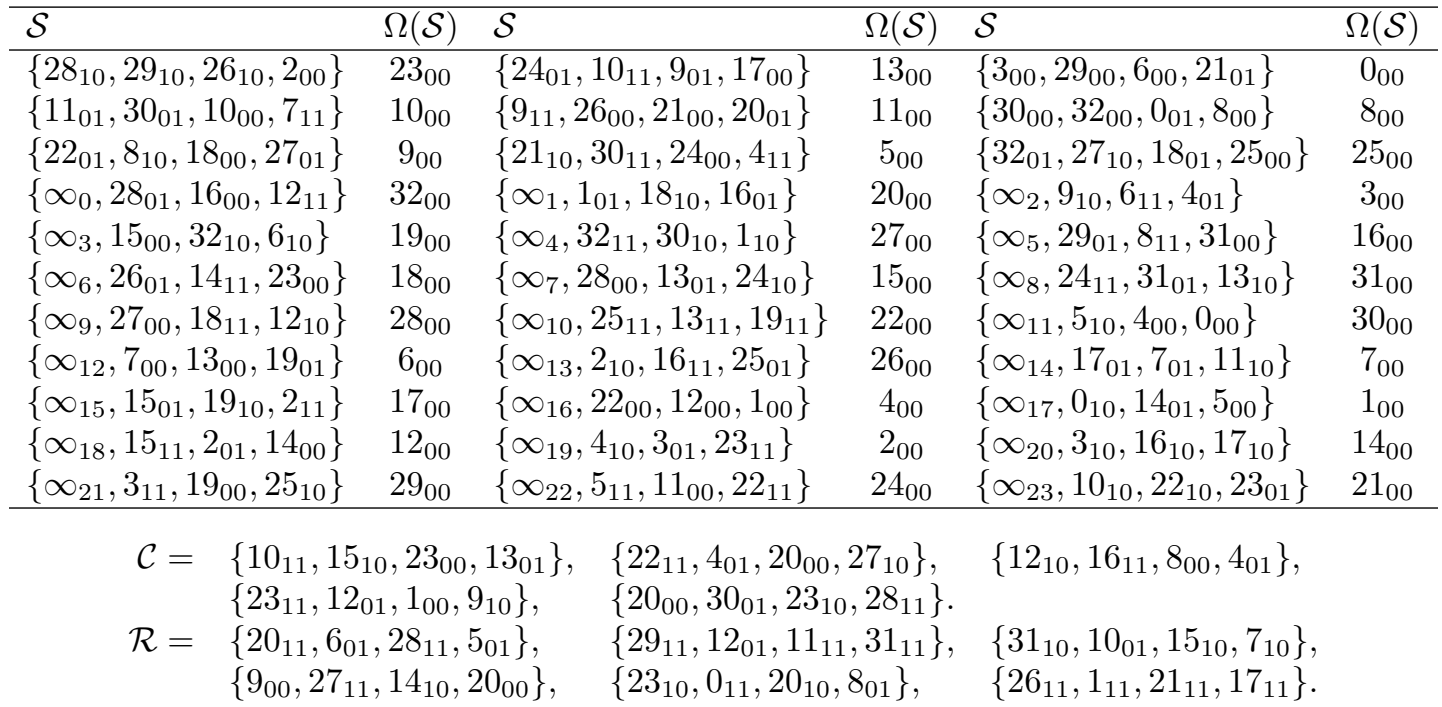

Proof of Theorem 2: We first construct a $\operatorname{GBTD}(4, m)$ for any $m \in N$, where $N=$ $\{28,32,33,34,37,38,39,44\}$.

For each $w \in\{5,6,7,8\}$, an $\operatorname{HGBTD}\left(4,4^{w}\right)$ is given by Yin et al. [12]. For each $m \in N$, apply Theorem 3.2, with IHGBTDs from Lemma 5, Lemma 6 and Lemma 7 and corresponding $\operatorname{HGBTD}\left(4,4^{w}\right)$ 's where $w \in\{5,6,7,8\}$ as ingredients, to produce the desired $\operatorname{HGBTD}\left(4,4^{m}\right)$. Hence, the desired $\operatorname{GBTD}(4, m)$ follows from Proposition 3.1.

Combining Proposition 1.1, Proposition 2.1 and Proposition 2.2, we complete the proof.

Acknowledgement We are grateful to the anonymous reviewers for their helpful comments.

\section{References}

[1] Y.M. Chee, H.M. Kiah, A.C.H. Ling, and C. Wang, Optimal equitable symbol weight codes for power line communications, Proceedings of the 2012 IEEE International Symposium on Information Theory, (2012), 671-675.

[2] C.J. Colbourn, J.H. Dinitz, The CRC Handbook of Combinatorial Designs, CRC Press, Boca Raton, FL, 2007.

[3] E.R. Lamken, Generalized balanced tournament designs, Trans. Amer. Math. Soc. 318 (1990), 473-490.

[4] E.R. Lamken, Existence results for generalized balanced tournament designs with block size 3, Des. Codes Cryptogr. 3 (1993), 33-61.

[5] R. C. Mullin and E. Nemeth, On furnishing Room squares, J. Combin. Theory 7 (1969) 266-272. 
[6] S. Niskanen and P. R. J. Östergård, Cliquer Users Guide, Version 1.0, Cliquer users guide, version 1.0, Tech. Report T48, Communications Laboratory, Helsinki University of Technology, 2003.

[7] A. Rosa and S.A. Vanstone, Starter-adder techniques for Kirkman squares and Kirkman cubes of small sides, Ars Combinatoria 14 (1982), 199-212.

[8] P.J.Schellenberg, G.H.J. Van Rees and S.A. Vanstone, The existence of balanced tournament designs, Ars Combinatoria 3 (1977), 303-318.

[9] N.V. Semakov, V.A. Zinov'ev, Equidistant $q$-ary codes with maximal distance and resolvable balanced incomplete block designs, Problemy Peredači Informacii, 4 (1968), $3-10$.

[10] J. Yan and J. Yin, Constructions of optimal $\operatorname{GDRP}(n, \lambda ; v)$ of type $\lambda^{1} \mu^{m-1}$, Discrete Appl. Math. 156 (2008), 2666-2678.

[11] J. Yan and J. Yin, A class of optimal constant composition codes from GDRPs, Des. Codes Cryptogr. 50 (2009), 61-76.

[12] J. Yin, J. Yan and C. Wang, Generalized balanced tournament designs and related codes, Des. Codes Cryptogr. 46 (2008), 211-230. 